\title{
LACRIMAL PLUGS AS A THERAPY FOR CONTACT LENS INTOLERANCE
}

\author{
TUULA VIRTANEN ${ }^{1}$, KAIJA HUOTARI ${ }^{2}$, MATTI HÄRKÖNEN ${ }^{2}$ and TIMO TERVO ${ }^{1}$ \\ Helsinki
}

\begin{abstract}
SUMMARY
We investigated whether canalicular occlusion with a lacrimal plug could increase objective and/or subjective comfort in contact lens $(C L)$ wearers $(n=9)$ with both a history of lens intolerance and relative tear fluid deficiency. A modified Schirmer test (MST) was performed with the lids closed and under topical anaesthesia. Patients with values of $\leqslant 10 \mathrm{~mm} / 5 \mathrm{~min}$ were included. The plugs were inserted into the inferior canaliculus of the eye with the lower MST result; the contralateral eye served as a control. Conjunctival hyperaemia, Rose-Bengal and fluorescein scores, as well as subjective irritation, decreased significantly in plugged eyes at the 1 month follow-up visit but had returned to the pre-operative level at the 3 month visit. Tear fluid plasmin activity was elevated prior to plug insertion. Plasmin activity of the plugged eye was significantly lower at the 1 month visit but neither tear fluid flow (in the collection capillary) nor plasmin release showed any changes. The mean MST value improved insignificantly, whereas conjunctival chemosis and limbal hyperaemia showed no improvement at the 1 or 3 month follow-up visits after inferior punctal occlusion. Occlusion of the lower canaliculus with a lacrimal plug seems to induce a relatively short-lasting subjective and objective benefit for $C L$ wearers. Plug escape or adaptive changes in lacrimal fluid secretion/ elimination rate probably took place during the followup. The association of increased plasmin activity with tear deficiency might lead to enhanced tissue proteolysis, and thus contribute to CL-related ocular surface changes.
\end{abstract}

Dry eye or ocular surface disease are the main causes of contact lens (CL) intolerance. ${ }^{1,2}$ Such patients also have an elevated risk for conjunctival or corneal inflammation or corneal neovascularisation., ${ }^{3,4}$ The ocular surface of patients with dry eye or ocular

From: ${ }^{1}$ Department of Ophthalmology and ${ }^{2}$ Department of Clinical Chemistry, Helsinki University Hospital, Helsinki, Finland.

Correspondence to: Dr Tuula Virtanen, University Eye Hospital, Haartmaninkatu 4 C, FIN-00290 Helsinki, Finland. surface disease is compromised not only by the inadequate infection barrier composed of intact tear film and corneal epithelium ${ }^{5}$ but also by factors associated with CL wear, of which oxygen deficiency, and allergic or toxic inflammatory reactions are probably the most important. ${ }^{6,7}$ Furthermore, the chemical $^{8}$ and physical ${ }^{9-11}$ properties of the tear fluid of dry eye patients show aberrations. Inadequate tear film may also reduce visual acuity. ${ }^{12}$

In recent years lacrimal plugs have been used to relieve dry eye symptoms. ${ }^{13-16}$ The use of lacrimal plugs also affects the composition of tear fluid by, for example, decreasing the hyperosmolality typical of dry eye. ${ }^{17}$

In the present study we investigated the subjective and objective effects of canalicular occlusion with lacrimal plugs in CL wearers with both lens intolerance and verified relative tear deficiency. The followup period after insertion of the plugs was 3 months. Tear fluid proteolytic activity due to plasmin ${ }^{18}$ was measured before and 1 month after plugging. It is known to be elevated in tears of CL wearers ${ }^{19-21}$ as well as to correlate with the tear fluid flow rate, ${ }^{18}$ and hence serves as an indicator of the tear-deficiencyrelated concentration effect of tear fluid components.

\section{Patients}

\section{MATERIALS AND METHODS}

Inclusion Criteria and Modified Schirmer Test (MST). The patients were referred primarily by an optician who normally monitored the patients. Each patient reported symptoms of $\mathrm{CL}$ intolerance, and the optician judged them to be related to tear deficiency and referred a total of $14 \mathrm{CL}$ wearers for further consultation. Patients with MST values of $\leqslant 10 \mathrm{~mm} / 5$ min were included. The technique, potential benefits and complications of punctal occlusion were carefully explained to the patients, who gave their written informed consent to participate in the study. After the study was completed the patients were allowed to choose whether not they wished the plug to be rinsed 
away (only one plug was removed). The study was also accepted by the ethics committee of Helsinki University Eye Hospital.

The MST was performed 5 minutes after application of the anaesthetic eye drop oxybuprocaine (Oculo-Fluresis, Leiras, Tampere, Finland) containing sodium fluorescein. The Schirmer test strips (Schirmer tear test, IOLAB Pharmaceuticals, Claremont, CA, USA) were placed in the temporal lower fornices of both eyes to avoid any corneal irritation. The patient was asked to hold the eyes closed in dim illumination for 5 minutes to avoid evaporation and mechanical irritation. The wetting (in millimetres) of the strips was then read.

Five of the original 14 patients had MST values over $10 \mathrm{~mm} / 5 \mathrm{~min}$, and their tear meniscus heights appeared normal at biomicroscopy; these individuals were not included in the present study.

The Subjects and Punctal Occlusion. Nine healthy CL users with MST results of $\leqslant 10 \mathrm{~mm} / 5 \mathrm{~min}$ at least in one eye were included in the present study. A Herrick no. 5 (8 patients) or no. 3 (1 patient) (Lacrimedics, Rialto, CA, USA) lacrimal plug was inserted into the lower canaliculus, whereafter symptoms and clinical findings associated with ocular surface disease were followed up for 3 months. The patients complained of no discomfort attributable to the plugs. The contralateral unplugged eyes served as controls. No history of any previous eye disease or ophthalmic surgery was reported by any of the selected $9 \mathrm{CL}$ wearers. Eight of the individuals were female and one was male. The mean age of the patients was 29.6 years (range 19-33 years). Eight of the subjects had daily-wear soft CLs and one had gas-permeable hard CLs. The mean CL wearing time was $8 \mathrm{~h} /$ day (range $4-12 \mathrm{~h} /$ day) and 5 times/week (range 3-6 times/week). The refractive correction varied from mild to moderate myopia (range -1.0 to $-6.0 \mathrm{D})$.

\section{Clinical Examinations}

The examination began by measurement of the visual acuity with the patient wearing the CLs. Tear fluid samples were taken before the remaining clinical procedures.

Ophthalmological Examinations. After collection of the tear fluid samples the individuals removed the CLs. The subjects were then examined again with a biomicroscope. Biomicroscopy of the anterior segment of the eye was performed to reveal any possible epithelial defect in the cornea or conjunctiva as well as signs of aqueous flare. The tarsal and bulbar conjunctivae were investigated for the presence of hyperaemia, follicles or papillae, and the anterior chamber for the presence of aqueous flare or inflammatory cells.
To reveal epithelial defects or alterations topical oxybuprocaine eye drops containing sodium fluorescein were applied into the lower fornices. The patient was then asked to blink a few times and viewed with a bio-microscope using blue light.

The intraocular pressure was subsequently measured with an applanation tonometer (Haag-Streit, Basel, Switzerland). The MST (see above) was performed 5 minutes later.

A small drop of Rose-Bengal (Rose Bengal Minims Eye Drops (ROS 1.0); Chauvin Pharmaceuticals, Romford, UK) stain was then applied on the lower fornix. Estimation of the Rose-Bengal score was done using the biomicroscope with standard illumination.

In the assessment of conjunctival hyperaemia and chemosis, limbal hyperaemia, and corneal RoseBengal or fluorescein staining, we used a scale of grades 0-1-2-3 (normal-mild-moderate-severe, respectively). In the estimation of subjective symptoms we use a scale of grades 0-1-2-3 (none-mildmoderate-severe, respectively) reported by the patients themselves on a modified Herrick questionnaire.

These examinations were repeated at the 1 and 3 month follow-up visits.

Tear Fluid Flow, Plasmin Activity and Plasmin Release. Tear fluid samples were collected for measurement of plasmin activity using a fluorometric assay. ${ }^{18}$ The result is expressed as international units/ litre (IU/l). Calibrated $5 \mu \mathrm{l}$ microcapillaries with blunted tips were used to aspirate $1-12 \mu l$ of tears from the lower fornix. The collection times $(\mathrm{min})$ and tear fluid flow rates in the collection capillary $(\mu \mathrm{l} /$ min) were measured. From these parameters the release of plasmin in tears (plasmin flux, $\mu \mathrm{IU} / \mathrm{min}$ ) was calculated as described earlier. ${ }^{18}$ The benefits and limitations of the procedure have been discussed previously. ${ }^{18}$ The samples were centrifuged immediately in Eppendorf tubes at $8000 \mathrm{~g}$ for 1 minute. The supernatants were aspirated, frozen on dry ice, and stored at $-75^{\circ} \mathrm{C}$ until assay. Since the sample volumes of some patients were very small, $5 \mu \mathrm{l}$ $0.9 \% \mathrm{NaCl}$ was added to the supernatants before assay and the results corrected accordingly.

The punctal occlusion, all ophthalmological examinations and tear fluid sample collection were performed by the same author (T.V.).

\section{RESULTS}

All patients had normal CL-corrected visual acuities of $1.0(20 / 20)$. The intraocular pressure was $<20$ $\mathrm{mmHg}$ in all cases; there were no signs of blepharitis, conjunctivitis or keratitis, or recognisable inflammation of the anterior chamber during any of the follow-up visits. 
Table I. Results of the modified Schirmer test in the dry eye and the contralateral control eye

\begin{tabular}{|c|c|c|c|c|c|c|}
\hline & \multicolumn{3}{|c|}{ Plugged eye } & \multicolumn{3}{|c|}{ Control eye } \\
\hline & 0 & 1 & 3 & 0 & 1 & 3 \\
\hline $\begin{array}{l}\text { Schirmer test } \\
p \text { value }^{\mathrm{a}}\end{array}$ & $50 \pm 0.8$ & $\begin{array}{c}88 \pm 2.1 \\
0.08\end{array}$ & $\begin{array}{c}8.2 \pm 2.9 \\
0.09\end{array}$ & $7.5 \pm 1.1$ & $7.2 \pm 1.8$ & $10.0 \pm 3.2$ \\
\hline
\end{tabular}

0 , at the first visit before insertion of the plug; 1 and 3 , at the 1 month and 3 month follow-up visits.

${ }^{a}$ Calculated using the Wilcoxon test.

Modified Schirmer Test. At the first visit before insertion of the plugs, the Schirmer test in the drier eyes was $2-10 \mathrm{~mm} / 5 \mathrm{~min}$ (mean $5.0 \pm 0.8 \mathrm{~mm} / 5 \mathrm{~min}$ ) and in the contralateral eyes $3-12 \mathrm{~mm} / 5 \mathrm{~min}$ (mean $7.5 \pm 1.1 \mathrm{~mm} / 5 \mathrm{~min}$ ). However, at the 1 month and 3 month follow-up visits no significant differences were noted in plugged compared with unplugged eyes (Table I). On the other hand, a tendency towards improvement could be observed in the plugged eye.

Conjunctival Hyperaemia, Conjunctival Chemosis and Limbal Hyperaemia. Conjunctival hyperaemia was significantly less intense in the plugged eyes at 1 month. At the 3 month visit this difference was no longer detectable. Conjunctival chemosis or limbal hyperaemia were no different between the plugged and unplugged eyes at the 1 or 3 month visits (Table II).

Rose-Bengal and Sodium Fluorescein Staining. The test scores were significantly better in the plugged eyes at 1 month. No differences were, however, found at the 3 month follow-up visit (Tables I, II).

Subjective Irritation and Itching. Subjective irritation was significantly reduced in the plugged eyes at the 1 month follow-up visit. However, at 3 months the patients reported no differences in irritation between the eyes; furthermore, differences no longer occurred between eyes with plugs versus eyes without them. The itching scores differed neither at 1 month nor 3 months (Table II).

Tear Fluid Flow, Plasmin Activity and Plasmin Release (Table III). There were no differences in tear fluid flow or plasmin release between the left and right eye before plug insertion or 1 month after it. Neither did tear fluid flow or plasmin release show significant differences at 1 month compared with the pre-operative values. However, plasmin activity decreased significantly $(p<0.01)$ in the plugged eyes (Wilcoxon signed rank test).

\section{DISCUSSION}

The Schirmer test, estimation of tear meniscus height, and Rose-Bengal and sodium fluorescein staining are probably the best and most widely used methods for assessment of the presence of ocular surface disease. The Schirmer test is known to have its limitations, while irritation of the eye and the resulting reflex tearing alters not only tear fluid flow rate but also the concentration of tear fluid components. ${ }^{22}$ Irritation can be minimised both by avoiding any contact between the strips and the cornea and by using topical anaesthesia. Values lower than $6 \mathrm{~mm} / 5$ min in the Schirmer I test give $85 \%$ sensitivity and $83 \%$ specificity for the detection of dry eye. ${ }^{23-25}$ Anaesthesia causes an approximately $20-30 \%$ drop in the value in the normal population. ${ }^{26}$ However, tear secretion is not linear but consists of cycles, each containing a peak, decay and 'final secretion rate'. ${ }^{26}$ Tear evaporation from both the ocular surface and the strip may also modify the results. ${ }^{26}$ On the other hand, evaporation of tears is subject to variation due to blinking frequency, tear fluid composition, CL wear, topical therapy and patient age. ${ }^{27}$ Although Holly $^{26}$ suggests that a well-controlled open eye Schirmer test is at least semiquantitative, we were also concerned about the great variations in evaporation rates and chose to perform a modified Schirmer test (MST) under topical anaesthesia (to avoid reflex tearing) and with the eyes closed (to avoid evaporation). We concluded that our patients did not have dry eye syndrome ${ }^{24}$ but probably only a relative tear

Table II. Objective and subjective findings of contact lens users $(n=9)$ with dry eyes before and after lacrimal plug therapy (mean \pm SEM)

\begin{tabular}{|c|c|c|c|c|c|c|c|c|}
\hline Eye & $\begin{array}{c}\text { Time } \\
\text { (months) }\end{array}$ & $\begin{array}{l}\text { Conjunctival } \\
\text { hyperaemia }\end{array}$ & $\begin{array}{l}\text { Conjunctival } \\
\text { chemosis }\end{array}$ & $\begin{array}{c}\text { Limbal } \\
\text { hyperaemia }\end{array}$ & Rose-Bengal & $\begin{array}{l}\text { Fluorescein } \\
\text { staining }\end{array}$ & $\begin{array}{l}\text { Subjective } \\
\text { irritation }\end{array}$ & Itching \\
\hline $\begin{array}{l}\text { Plugged eye } \\
\text { Control eye }\end{array}$ & 0 & $\begin{array}{l}1.3 \pm 0.2 \\
1.2 \pm 0.2\end{array}$ & $\begin{array}{l}1.1 \pm 0.3 \\
1.1 \pm 0.3\end{array}$ & $\begin{array}{l}1.2 \pm 0.3 \\
1.1 \pm 0.3\end{array}$ & $\begin{array}{l}0.9 \pm 0.2 \\
1.0 \pm 0.0\end{array}$ & $\begin{array}{l}0.9 \pm 0.2 \\
0.7 \pm 0.2\end{array}$ & $\begin{array}{l}2.1 \pm 0.3 \\
1.8 \pm 0.3\end{array}$ & $\begin{array}{l}0.2 \pm 0.2 \\
0.2 \pm 0.2\end{array}$ \\
\hline $\begin{array}{l}\text { Plugged eye } \\
p \text { value }^{\mathrm{a}} \\
\text { Control eye }\end{array}$ & 1 & $\begin{array}{c}0.8 \pm 0.2 \\
0.05 \\
1.2 \pm 0.3\end{array}$ & $\begin{array}{c}0.7 \pm 0.2 \\
0.62 \\
1.0 \pm 0.2\end{array}$ & $\begin{array}{c}0.8 \pm 0.2 \\
1.00 \\
0.9 \pm 0.3\end{array}$ & $\begin{array}{c}0.6 \pm 0.2 \\
0.03 \\
1.1 \pm 0.2\end{array}$ & $\begin{array}{c}0.2 \pm 0.2 \\
0.002 \\
0.9 \pm 0.1\end{array}$ & $\begin{array}{c}0.8 \pm 0.3 \\
0.05 \\
1.2 \pm 0.3\end{array}$ & $\begin{array}{c}0.1 \pm 0.1 \\
1.00 \\
0.2 \pm 0.2\end{array}$ \\
\hline $\begin{array}{l}\text { Plugged eye } \\
p \text { value }^{\mathrm{a}} \\
\text { Control eye }\end{array}$ & 3 & $\begin{array}{c}0.9 \pm 0.2 \\
1.00 \\
0.9 \pm 0.2\end{array}$ & $\begin{array}{c}0.9 \pm 0.3 \\
1.00 \\
1.0 \pm 0.2\end{array}$ & $\begin{array}{c}0.4 \pm 0.2 \\
1.00 \\
0.4 \pm 0.2\end{array}$ & $\begin{array}{c}0.4 \pm 0.2 \\
1.00 \\
0.6 \pm 0.2\end{array}$ & $\begin{array}{c}0.2 \pm 0.2 \\
0.67 \\
0.3 \pm 0.2\end{array}$ & $\begin{array}{c}1.1 \pm 0.3 \\
0.15 \\
1.2 \pm 0.3\end{array}$ & $\begin{array}{c}0.3 \pm 0.2 \\
1.00 \\
0.3 \pm 0.2\end{array}$ \\
\hline
\end{tabular}

${ }^{\mathrm{a} C a l c u l a t e d}$ using Fisher's square test. 
Table III. Tear fluid plasmin-like activity in eyes with and without a lacrimal plug

\begin{tabular}{lcccc}
\hline & Time & $\begin{array}{c}\text { Tear fluid flow } \\
(\mu \mathrm{l} / \mathrm{min})\end{array}$ & $\begin{array}{c}\text { Plasmin activity } \\
(\mathrm{IU} / \mathrm{l})\end{array}$ & $\begin{array}{c}\text { Plasmin flux } \\
(\mu \mathrm{IU} / \mathrm{min})\end{array}$ \\
\hline $\begin{array}{l}\text { Plugged eye } \\
\text { Conrol eye }\end{array}$ & Day 0 & $1.8 \pm 0.6$ & $11.0 \pm 1.7$ & $15.0 \pm 4.6$ \\
$p$ value $^{\mathrm{a}}$ & & $2.2 \pm 0.8$ & $9.2 \pm 1.5$ & $14.9 \pm 4.6$ \\
$\begin{array}{l}\text { Plugged eye } \\
\text { Control eye }\end{array}$ & $\mathrm{NS}$ & $\mathrm{NS}$ & $\mathrm{NS}$ \\
$p$ value $^{\mathrm{a}}$ & 1 month & $2.7 \pm 0.9$ & $6.4 \pm 2.0$ & $11.2 \pm 2.8$ \\
$p$ value $^{\mathrm{b}}$ & & $2.3 \pm 0.7$ & $10.2 \pm 3.8$ & $\mathrm{NS}$ \\
\hline
\end{tabular}

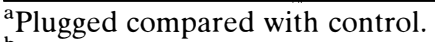

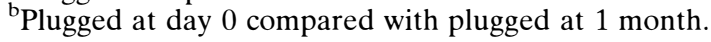

deficiency, which, however, presumbly accounted for their CL intolerance. A closed eye Schirmer test has previously been used and recommended by others. ${ }^{28}$

CLs are known to compromise the lipid layer in the tear fluid, leading to increased tear fluid evaporation, especially in an environment with low humidity. ${ }^{29}$ Cessation of CL wear is usually an adequate therapy, but many patients would like to continue lens wear.

Our relatively small study ( 9 patients) confirms the short-term beneficial effect of canalicular occlusion for CL wearers. ${ }^{16}$ Giovagnoli and Graham ${ }^{16}$ inserted removable Freeman silicone plugs for 21 patients. They found that during the follow-up period of only 3 weeks the plug users were subjectively more comfortable. Both our objective findings (conjunctival hyperaemia, Rose-Bengal and fluorescein scores) and the subjective symptoms reported by the patients also initially improved, as shown by the results at the 1 month follow-up visit.

Our study can be criticised because of the fact the patients knew which one of the canaliculi had been occluded. However, while the benefit of the procedure was no longer detectable at the 3 month followup visit, bias due to patients is unlikely and other explanations should be sought. First, since the Herrick plugs can neither be visualised like Eagle plugs (Eagle Vision, Memphis, TN, USA) nor are they radio-opaque, we could not exclude the possibility of plug escape. This could be demonstrated by dacryoscintigraphy. ${ }^{30}$ The manufacturer is probably also concerned by this possibility, and now recommends the use of a larger Herrick size 7 plug instead of the size 5 or 3 used in our study. Secondly, Doane $^{31}$ described that the upper canaliculus has the potential to dry many more tears than it normally does. Hence, adaptive changes may take place after occlusion of the lower canaliculus, resulting in enhanced elimination of tears via the upper canaliculus. The manufacturer now recommends that the plug should first be inserted in the upper canaliculus (Herrick R.S., personal communication to T.T. 1996). This measure also indirectly supports our findings.

Short-term (up to 7 days) occlusion of both upper and lower canaliculi increases tear fluid stability and meniscus height. ${ }^{32}$ Hence the observed decrease in the tear fluid plasmin activity in the plugged eye at 1 month was to be expected. Enrolment of a larger number of patients would probably have made it possible to verify an improvement in the MST results as well. This was not considered important, because of the observed relatively rapid loss of therapeutic efficacy.

Tear fluid plasmin activity reflects the welfare of the ocular surface; mild irritation such as the Schirmer test $^{33}$ or CL wear, ${ }^{19-21}$ and especially various corneal and conjunctival diseases, ${ }^{34,35}$ increase tear fluid plasmin activity. We noted that is essential to measure the tear fluid flow to correct the plasmin activity results obtained. ${ }^{18}$ In this way those patients with relatively low tear fluid flow were found to have higher plasmin activities, whereas the plasmin release levels were virtually the same. These findings were confirmed in the present study. Tear fluid mean plasmin activities were about 3-4 times higher than those of control individuals ${ }^{18}$ twice as high as those of control individuals with mild tear deficiency $^{18}$ and 4-5 times higher than of those normal asymptomatic soft $\mathrm{CL}$ wearers ${ }^{21}$ in our earlier studies. However, plasmin release in tears was lower in this patient group than it was in the tears of asymptomatic CL wearers. ${ }^{18} \mathrm{We}$ have also shown that during uncomplicated healing of human photorefractive keratectomy wounds the plasmin activity actually decreases due to reflex tearing despite the remarkably increased plasmin release, ${ }^{36}$ which further underlines the dependency of plasmin activity on the tear fluid flow rate.

In conclusion, enhancement of precorneal tear film of $\mathrm{CL}$ wearers by canalicular occlusion might facilitate continuation of lens wear in patients with $\mathrm{CL}$ intolerance due to inadequate quantity or quality of precorneal tear film. Punctal occlusion with removable plugs may restore the normal tear fluid composition and thus prevent excessive tissue proteolysis or allow a longer persistence of growth factors on the ocular surface. The technique used in our study, however, needs to be improved to achieve a reasonably long-lasting clinical response. Visible 
plugs that are easily monitored, and occlusion of the upper canaliculus first and then (if required) both canaliculi, might give a better therapeutic result. This, however, remains to be studied in a larger controlled study.

The present study was supported by the Finnish Medical Council and the Scientific Foundation of Instrumentarium Co.

Key words: Lacrimal plugs, Contact lens, Tear deficiency, Tear fluid, Plasmin activity.

\section{REFERENCES}

1. Farris R. The dry eye: its mechanisms and therapy, with evidence that contact lens is a cause. CLAO $\mathbf{j}$ 1986;12:234-46.

2. Holly F. Tear film physiology and contact lens wear. II. Contact lens-tear film interactions. Am J Optom Physiol Optics 1981;58:331-41.

3. Chalupa E, Swarbrick H, Holden B, Sjöstrand J. Severe corneal infections associated with contact lens wear. Ophthalmology 1987;94:17-22.

4. Arentsen J. Corneal neovascularisation in contact lens wearers. Int Ophthalmol Clin 1986;26:15-23.

5. Holly F, Lemp M. Tear physiology and dry eyes. Surv Ophthalmol 1977;22:69-87.

6. Farris R. Tear analysis in contact lens wearers. Trans Am Ophthalmol Soc 1985;88:501-45.

7. Holly F. Surface chemistry of contact lens wear. In: Holly FJ, Lemp MA, editors. The preocular tear film and dry eye syndromes. Int Ophthalmol Clin 13. Boston: Little and Brown, 1973:279-86.

8. Holly F, Lemp M. Surface chemistry of the tear film: implications for dry eye syndromes, contact lenses, and ophthalmic polymers. Contact Lens Soc Am J 1971; 5:12-9.

9. De Roetth A. Low flow of tears: the dry eye. Am J Ophthalmol 1952;35:782-7.

10. Tapaszto I. Pathophysiology of human tears: the preocular tear film and dry eye syndromes. Int Ophthalmol Clin 1973;13:119-22.

11. Schirmer O. Studies on the physiology and pathology of the secretion and drainage of tears. Graefes Arch Klin Ophthalmol 1903;56:197-291.

12. Reiger G. The importance of precorneal tear film for the quality of optical imaging. $\mathrm{Br} \mathrm{J}$ Ophthalmol 1992;76:157-8.

13. Freeman J. The punctum plug: evaluation of the new treatment for the dry eye. Trans Am Acad Ophthalmol Otolaryngol 1975;79:OP 874-9.

14. Adams A. Silicone plug for punctal occlusion. Trans Ophthalmol Soc UK 1978;98:499.

15. Willis R, Folberg R, Krachmer J, Holland E. The treatment of aqueous deficient dry eye with removable punctal plugs: a clinical and impression-cytologic study. Ophthalmology 1987;94:514-8.

16. Giovagnoli D, Graham S. Inferior punctal occlusion with removable silicone punctal plugs in the treatment of dry-eye related contact lens discomfort. J Am Optom Assoc 1992;63:481-5.

17. Gilbard J, Farris R, Santamaria J. Osmolarity of tear microvolumes in keratoconjunctivitis sicca. Arch Ophthalmol 1978;96:677-81.

18. Tervo T, Honkanen N, van Setten G-B, Virtanen T,
Tarrkanen A, Härkönen M. Fluorometric assay for determination of tear fluid plasmin activity. Cornea 1994;13:148-55.

19. van Setten G-B, Tervo T, Andersson R, Perheentupa J, Tarkkanen A. Plasmin and epidermal growth factor in the tear fluid of contact-lens wearers: effect of wearing different types of contact lenses and association with clinical findings. Ophthalmic Res 1990;22:233-40.

20. Vannas A, Sweeney D, Holden B, Sapyska E, Salonen E, Vaheri A. Tear plasmin activity with contact lens wear. Curr Eye Res 1992;11:243-51.

21. Virtanen T, Honkanen N, Härkönen M, Tarkkanen A, Tervo T. Elevation of tear fluid plasmin activity of contact lens wearers studied with a rapid fluorometric assay. Cornea 1994;13:210-3.

22. Fullard RJ, Tucher D. Tear protein composition and the effects of stimulus. In: Sullivan DA, editor. Lacrimal gland, tear film, and dry eye syndromes. Adv Exp Med Biol 1994;350:309-14.

23. van Bijsterveld OP. Diagnostic tests in the sicca syndrome. Arch Ophthalmol 1969;82:10-4.

24. Bron AJ, Mengher LS. The ocular surface in keratoconjunctivitis sicca. Eye 1989;3:428-37.

25. Mackie IA, Seal DV. The questionably dry eye. Br J Ophthalmol 1981;65:2-9.

26. Holly FJ. Lacrimation kinetics as determined by a Schirmer-type technique. In: Sullivan DA, editor. Lacrimal gland, tear film and dry eye syndromes: basic science and clinical relevance. Adv Exp Med Biol 1994;350:543-8.

27. Tomlinson A, Giesbrecht C. Effect of age on human tear film evaporation in normals. In: Sullivan DA, editor. Lacrimal gland, tear film, and dry eye syndromes. Adv Exp Med Biol 1994;350:271-4.

28. Prause JU, Frost-Larsen K, Isager H, Manthorpe R. Tear absorption into the filter-paper strip used in the Schirmer-I-test. Acta Ophthalmol (Copenh) 1982; 60:70-8.

29. Tomlinson A, Cedarstaff TH. Tear evaporation from the human eye: the effects of contact lens wear. $\mathrm{J} \mathrm{Br}$ CL Assoc 1982;5:141-50.

30. Denffer H, Dressler J, Pabst H. Lacrimal dacryoscintigraphy. Semin Nucl Med 1989;14:8-15.

31. Doane MG. Blinking and the mechanics of the lacrimal drainage system. Ophthalmology 1981;88:844-50.

32. Patel S, Grierson D. Effect of collagen punctal occlusion on tear stability and volume. In: Sullivan DA, editor. Lacrimal gland, tear film and dry eye syndromes: basic science and clinical relevance. Adv Exp Med Biol 1994;350:605-8.

33. van Setten G-B, Stephens R, Tervo T, Salonen E-M, Tarkkanen A, Vaheri A. Effects of Schirmer test on the fibrinolytic system in the tear fluid. Exp Eye Res 1990;50:135-41.

34. Berman M, Leary R, Gage J. Evidence for a role of the plasminogen activator-plasmin system in corneal ulceration. Invest Ophthalmol Vis Sci 1980;19:1204-21.

35. Salonen E-M, Tervo T, Törmä E. Tarkkanen A, Vaheri A. Plasmin in tear fluid of patients with corneal ulcers: basis for a new therapy. Acta Ophthalmol (Copenh) 1987;65:3-12.

36. Tervo T, Virtanen T, Honkanen N, Härkönen M, Tarkkanen A. Tear fluid plasmin activity after excimer laser photorefractive keratectomy. Invest Ophthalmol Vis Sci 1994;35:3045-50. 